\title{
LA PROTECCIÓN AL MEDIO AMBIENTE EN EL SECTOR HIDROCARBUROS EN MÉXICO COMO ELEMENTO TRANSFORMADOR DE LA AGENDA INTERNACIONAL DE SEGURIDAD INDUSTRIAL 2030, EN ATENCIÓN A LA TUTELA EFECTIVA DE LOS DESCA EN EL ESTADO DE NUEVO LEÓN
}

\author{
THE PROTECTION TO THE ENVIRONMENT IN THE SECTOR HYDROCARBONS IN \\ MÉXICO LIKE ELEMENT TRANSFORMER OF THE SCHEDULE INTERNACIONAL OF \\ SECURITY INDUSTRIAL 2030, IN ATTENTION TO GUARDIANSHIP EFFECTIVE OF THE \\ REST IN THE STATE OF NUEVO LEÓN.
}

José Luis Leal Espinoza

Doctor en Filosofía (PhD) con especialidad en Derecho Constitucional y derechos fundamentales por la Universidad de Alicante, España. Especialista en Justicia Constitucional y Argumentación Jurídica por la Universidad de Castilla La Mancha, España. Catedrático Investigador a Tiempo Completo y Vicedecano de Investigación y Posgrado de la Facultad de Ciencias Políticas y Sociales de la Universidad Autónoma de Coahuila, México. Miembro del Sistema Nacional de Investigadores Nivel I del Consejo Nacional de Ciencia y Tecnología de México. Miembro Numerario del Instituto Iberoamericano de Estudios Constitucionales, España. Asesor Jurídico de la LXIV Legislatura de la Cámara de Diputados del H. Congreso de la Unión, México. Correo electrónico: jose.leal@uadec.edu.mx

Convidado

RESÚMEN: Actualmente, México afronta un problema serio con el Ducto Net que transporta Gas Natural desde la frontera con Estados Unidos hasta el Ducto Los Ramones. Este ducto tiene 35 años en operación continua, y requiere de mantenimientos para asegurar el correcto Transporte de Gas Natural. Esta situación pone a relieve la escasa infraestructura que tiene México y denota el área de oportunidad por la falta de planeación estratégica para solventar y prevenir estos problemas que suelen presentarse al momento de implementar los programas de mantenimiento de los proyectos que se encuentran activos; ya que, al ser el único Ducto de Gas Natural que abastece esa zona, implica consecuentemente negarle a los gobernados el abastecimiento necesario de este Hidrocarburo que se considera fundamental para su uso en las Familias, así como en la Industria.

Palavras-chave: Sector energético. Hidrocarburos. Tutela efectiva. Medio ambiente. Derechos humanos.

\begin{abstract}
México is currently facing a serious problem with the Net Pipeline that transports Natural Gas from the US border to the Los Ramones Pipeline. This pipeline has been operating for the last 35 years and requires maintenance to ensure the correct Transportation of Natural Gas. This situation highlights the poor infrastructure that Mexico has and denotes the area of opportunity due to the lack of strategic planning to solve and prevent these problems that usually arise when implementing the maintenance programs of the projects that are active; since, being the only
\end{abstract}


Natural Gas Pipeline that supplies that area, it consequently implies denying the governed the necessary supply of this Hydrocarbon that is considered fundamental for its use in Families, as well as in Industry.

Keywords: Energy sector. Hydrocarbons. Effective protection. Environment. Human rights.

SUMÁRIO: Introducción. Conclusiones. Referencias

\section{INTRODUCCIÓN}

En la actualidad los avances tecnológicos para llevar a cabo los programas de mantenimiento en Ductos, son muy avanzados, existiendo técnicas especializadas como el "Hot Tapping" que permite realizar mantenimientos mayores y menores en ductos de alta y baja presión sin necesidad de parar o disminuir el flujo de la molécula que se está transportando, es una técnica de fijación de un accesorio de ramificación mecánica o soldada a la tubería o equipo en servicio, y la creación de una abertura en la tubería o el equipo por la perforación o el corte de una porción de la tubería o equipo dentro de la instalación adjunta. El Hot Tapping se realiza generalmente cuando no es posible poner el equipo o la tubería fuera de servicio, para purgar o limpiar por métodos convencionales .

De igual forma también existiría una menor recaudación por parte de la Secretaría de Hacienda y Crédito Público y un incremento en el costo, siendo afectados tanto el gobernante como el gobernado. Simultáneamente el Estado se encuentra construyendo un Ducto el cual todavía no se encuentra terminado y en condiciones de operar, por lo que para minimizar la problemática de abastecimiento, recaudación y fomento a la inversión, se requiere de un análisis a fondo respecto al tipo de Riesgo detectado en el Ducto, comparándolo con la información proporcionada en su Sistema de Administración de Riesgos, para que por medio de una ecuación se derive la probabilidad exacta de la ocurrencia de un evento indeseable, medido en términos de las consecuencias o afectaciones que pudieran presentarse en las personas, instalaciones, medio ambiente o en la comunidad.

México se encuentra construyendo un mejor futuro, a partir de la reforma energética que se publicó en el Diario Oficial de la Federación el 20 de diciembre 2013 en el Decreto por el que se reforman y adicionan diversas disposiciones de la Constitución Política de los Estados Unidos Mexicanos en Materia Energética, a través del cual se publicaron 21 artículos en el apartado de transitorios referentes al tema, que permitieron crear los Órganos Regulados Coordinados en Materia Energética, encargados de velar por el buen funcionamiento de la red de Ductos en el tema de Hidrocarburos, específicamente en este caso Gas Natural, así como como ampliar la capacidad jurídica de los Órganos que intervienen en la revisión de contratos, el otorgamiento de permisos y la emisión de Regulación que permita llevar a cabo estas actividades sin perjuicio de las personas, el medio ambiente o las instalaciones.

Para efecto de analizar y realizar un análisis jurídico a fondo sobre la actual situación de este tendido de Ductos de Transporte de Gas Natural, se requerirían de mayores elementos técnicos e información detallada respecto a los resultados de la verificación y auditoria del Ductos, en el cual se detectaron los Riesgos que menciona el CENAGAS, así como los datos respecto a la situación actual del Ducto, los tipos de mantenimientos efectuados, el resultado de las auditorías internas y externas del SASISOPA, así como el Análisis de Riesgos de la totalidad del proyecto al momento del inicio de operaciones.

Sin embargo, basándonos en la información pública y partiendo del deber ser con apego a derecho, es preciso mencionar que, para llevar a cabo las operaciones de Transporte por Ducto de Gas Natural, se han publicado diversas regulaciones en el Diario Oficial de la Federación, respecto a los temas más críticos en la Seguridad Operativa y salvaguarda del medio ambiente,

Revista de Direito Brasileira | Florianópolis, SC | v. 26 | n. 10 | p. 230-242 | Mai./Ago. 2020 
mismas que son de observancia obligatoria para todos los permisionados que realizan actividades del Sector Hidrocarburos.

La Agencia Nacional de Seguridad Industrial y de protección al medio ambiente del Sector Hidrocarburos, tiene por objeto la protección de las personas, el medio ambiente y las instalaciones del Sector, mismas que son descritas por el orden de importancia de las mismas, por lo tanto, aporta los elementos técnicos en relación a la Seguridad Industrial, Seguridad Operativa y Protección Ambiental (SISOPA) en procuración de la política energética y ambiental del País, así como para la formulación de los programas sectoriales en estas materias, misma que cuenta con atribuciones para Regular, Supervisar y Sancionar a los permisionados que no cumplan con la Regulación emitida.

En este orden de ideas, la ASEA publicó la Norma Oficial Mexicana NOM-007-ASEA2016, Transporte de gas natural, etano y gas asociado al carbón mineral por medio de ductos, misma que hace referencia a que los Regulados que realizan la actividad de Transporte de Gas Natural deberán obtener un Dictamen de Operación y Mantenimiento de forma anual, mismo que será emitido por un Tercero Autorizado por Agencia, asimismo, establece los requisitos de mantenimiento de cada Ducto, y que estos deben ser acordes a los especificados en el SASISOPA de cada permisionado, este es auditable y verificable por la misma ASEA, en este sentido, si la Agencia detecta que se omitieron mantenimientos preventivos o correctivos, pudiera parecer a una sanción e incluso a la clausura del tendido de Ductos.

Por su parte, en ejercicio de sus atribuciones, el Centro Nacional del Control de Gas (CENAGAS), encargado de la operación del Sistema Nacional de Ductos de Transporte y Almacenamiento, mismo que requiere a quienes realizan las actividades de Transporte $\mathrm{y}$ Distribución, vigilar, rehabilitar y dar mantenimiento a la red de Ductos, hizo de conocimiento público que Ducto Net en diversos tramos de Reynosa, Monterrey, Torreón y Chihuahua requiere de atención, pues según el CENAGAS se detectaron condiciones de peligro para la población, el medio ambiente y las Instalaciones del Transporte de Hidrocarburos por medio Ductos. En casi 666 kilómetros presentan Riesgos altos y 8 mil 166 kilómetros enfrentan Riesgos medios. En los diagnósticos de los análisis costo-beneficio de cada uno de los cuatro proyectos de inversión, el CENAGAS hizo hincapié en que en esos Ductos para el Transporte de Gas fueron detectados serios problemas de Mantenimiento Integral de los Sistemas de Ductos para Gas Natural, pues según la información que presenta este organismo, la red de ductos cuenta con más 35 años continuos de operación, por lo que es necesario actualizar las Instalaciones para que sean acordes al programa Rehabilitación y Mantenimiento de Infraestructura de Ductos a Nivel Nacional.

Por otra parte la Secretaria del Medio Ambiente y Recursos Naturales (SEMARNAT), tiene participación conjunta con la ASEA, ya que ambos se encargan de salvaguardar las condiciones de protección ambiental de los suelos, flora y fauna silvestres a que se sujetan las actividades del Transporte de Hidrocarburos, ello para evitar o minimizar las alteraciones ambientales que pudieran generarse por Incidentes o Accidentes en las operaciones del Ducto, así como la prevención de derrames y fugas de Hidrocarburos que pudieran dar como resultado una alteración al ambiente que derive en la necesidad de una remediación de los suelos, derivada de las afectaciones en que su caso, por falta de los mantenimientos necesarios pudieran derivarse.

Ahora bien, no se puede perder de vista que el gobierno tiene el deber de velar por el bien común, la vida y la seguridad de sus ciudadanos, a través de diversos órganos encargados de velar por la seguridad del Sector, se deben analizar y toman las decisiones pertinentes en los temas estratégicos de la reforma energética en materia de seguridad, recaudación y a efecto de garantizar, proteger, promover y garantizar los derechos humanos a un medio ambiente sano.

Manifestado lo anterior, podemos advertir que los órganos competentes para emitir una valoración sobre la factibilidad de la bajar la capacidad volumétrica del transporte de Gas Natural en ese Ducto, serían la ASEA y CENAGAS en coordinación con la Secretaría de Economía, sin embargo, para poder sustentar un análisis jurídico de fondo, se requeriría de mayor información 
detallada de las condiciones actuales del ducto y los tipos de mantenimiento que le fueron requeridos.

En conclusión, en la realización de las actividades del Sector Hidrocarburos, una de las principales enmiendas de los órganos reguladores, es velar por la Seguridad en los procesos dentro de todas las etapas de la cadena de valores, ya que la regulación que actualmente se encuentra publicada, nos indica que no hay factor más importante a proteger que la vida y el derecho a gozar de un medio ambiente sano, esto solo podrá ser logrado bajo la cultura de prevención y concientización de los Riesgos que conlleva cada proceso en del Sector, por lo que es de suma importancia exigir a todos los Regulados el debido cumplimiento de la regulación emitida.

La sultana del norte ha resentido la falta de presupuesto y un nuevo marco jurídico que mejore la inspección de empresas que inciden sobre la contaminación, las y los ciudadanos han demandado acciones a las distintas instancias para atacar este fenómeno.

El estado de Nuevo León ha sido testigo de un alto grado de desarrollo económico y urbano, tan solo durante los últimos 4 años ha recibido 11 mil 484 millones de dólares de Inversión Extranjera Directa. Sabemos que las zonas urbanas concentran la mayor parte de la población así como las actividades que detonan el desarrollo, no obstante, no podemos prescindir de los problemas que este proceso conlleva, principalmente el deterioro ambiental, lo cual impacta de manera significativa en la salud y calidad de vida de los ciudadanos.

Actualmente la población neolonesa asciende a 5.3 millones de habitantes, de los cuales el $85 \%$ se concentra en la ZMM. Durante los últimos quince años se ha intensificado la urbanización de la Zona Metropolitana de Monterrey (ZMM), que involucra a nueve municipios.

ESPECIFICACIONES ADICIONALES DE GASOLINAS POR REGIÓN

\begin{tabular}{|c|c|c|c|c|c|c|c|}
\hline \multirow{3}{*}{ Propiedad } & \multirow{3}{*}{$\begin{array}{c}\text { Unida } \\
\text { d }\end{array}$} & \multirow{3}{*}{ Método de prueba } & \multicolumn{5}{|c|}{ Valor límite } \\
\hline & & & $\underset{\mathbf{M}}{\mathbf{Z M V}}$ & ZMG & ZMM & Resto & el País \\
\hline & & & \multicolumn{3}{|c|}{$\begin{array}{c}\text { Gasolinas Premium y } \\
\text { Regular }\end{array}$} & $\begin{array}{c}\text { Gasolina } \\
\text { Premiu } \\
\text { m }\end{array}$ & $\begin{array}{c}\text { Gasolin } \\
\quad \mathbf{a} \\
\text { Regular }\end{array}$ \\
\hline Aromáticos & $\%$ vol. & $\begin{array}{l}\text { Tipos de hidrocarburos } \\
\text { en productos líquidos } \\
\text { de petróleo por } \\
\text { absorción de indicador } \\
\text { fluorescente } \\
\text { (ASTM D1319) }\end{array}$ & $\begin{array}{l}25.0 \\
\text { máxim } \\
\text { o }\end{array}$ & $\begin{array}{c}32.0 \\
\text { máxim } \\
\text { o } \\
25.0 \\
\text { máxim } \\
\text { o a } \\
\text { partir } \\
\text { del } 1^{\circ} \\
\text { de } \\
\text { enero } \\
\text { de } \\
2020\end{array}$ & $\begin{array}{c}32.0 \\
\text { máxim } \\
\text { o } \\
25.0 \\
\text { máxim } \\
\text { o a } \\
\text { partir } \\
\text { del } 1^{\circ} \\
\text { de } \\
\text { enero } \\
\text { de } 2020\end{array}$ & $\begin{array}{c}32.0 \\
\text { máximo }\end{array}$ & Informar \\
\hline Olefinas & $\%$ vol. & $\begin{array}{l}\text { Tipos de hidrocarburos } \\
\text { en productos líquidos } \\
\text { de petróleo por } \\
\text { absorción de indicador } \\
\text { fluorescente } \\
\text { (ASTM D1319) }\end{array}$ & $\begin{array}{c}10.0 \\
\text { máxim } \\
\text { o }\end{array}$ & $\begin{array}{c}11.9 \\
\text { máxim } \\
\text { o } \\
10.0 \\
\text { máxim } \\
\text { o a } \\
\text { partir }\end{array}$ & $\begin{array}{c}11.9 \\
\text { máxim } \\
\text { o } \\
10.0 \\
\text { máxim } \\
\text { o a } \\
\text { partir }\end{array}$ & $\begin{array}{c}12.5 \\
\text { máximo }\end{array}$ & Informar \\
\hline
\end{tabular}




\begin{tabular}{|c|c|c|c|c|c|c|c|}
\hline & & & & $\begin{array}{c}\text { del } 1^{\circ} \\
\text { de } \\
\text { enero } \\
\text { de } \\
2020\end{array}$ & $\begin{array}{c}\text { del } 1^{\circ} \\
\text { de } \\
\text { enero } \\
\text { de } 2020\end{array}$ & & \\
\hline Benceno & $\%$ vol. & $\begin{array}{l}\text { Determinación de } \\
\text { benceno y tolueno en } \\
\text { gasolina terminada para } \\
\text { uso en motores y } \\
\text { aviación por } \\
\text { cromatografía de gases } \\
\text { (ASTM D3606, D5580, } \\
\text { D6277) }\end{array}$ & $\begin{array}{l}1.0 \\
\text { máxim } \\
\text { o }\end{array}$ & $\begin{array}{c}1.0 \\
\text { máxim } \\
\text { o }\end{array}$ & $\begin{array}{l}1.0 \\
\text { máxim } \\
\text { o }\end{array}$ & $\begin{array}{l}2.0 \\
\text { máximo }\end{array}$ & $\begin{array}{c}2.0 \\
\text { máximo }\end{array}$ \\
\hline Azufre total & $\mathrm{mg} / \mathrm{kg}$ & $\begin{array}{l}\text { Determinación de } \\
\text { azufre total en } \\
\text { hidrocarburos ligeros } \\
\text { (ASTM D5453, D2622, } \\
\text { D7039, D7220) }\end{array}$ & \multicolumn{5}{|c|}{$\begin{array}{l}30 \text { promedio }^{(1)} \\
80 \text { máximo }(2)\end{array}$} \\
\hline Oxígeno $\left.3^{(4)}\right)^{(5)} 6$ & $\%$ masa & $\begin{array}{lr}\text { Determinación } & \text { de } \\
\text { MTBE, ETBE, } & \text { TAME, DIPE y } \\
\text { alcoholes hasta C4 en } \\
\text { gasolinas } & \text { por } \\
\text { cromatografía de gases } \\
\text { (ASTM D4815) } \\
\text { Determinación de } \\
\text { MTBE, r ETBE, } \\
\text { TAME,DIPE, etanol y } \\
\text { terbutanol en gasolinas } \\
\text { por espectros copia } \\
\text { infrarroja } \\
\text { (ASTM D5845) }\end{array}$ & \multicolumn{3}{|c|}{ 1.0â2.7 máximo. } & \multicolumn{2}{|c|}{2.7 máximo } \\
\hline
\end{tabular}

Dicho lo anterior, resulta imperante tomar acciones que contribuyan a equilibrar el desarrollo de la entidad --principalmente en la Zona Metropolitana de Monterrey-- con la conservación ambiental. Basta con revisar el marco normativo para darnos cuenta que es una preocupación que ha trascendido fronteras y la Federación tiene la responsabilidad de tomar parte.

En los últimos años, el derecho internacional ha tomado conciencia acerca de la problemática que existe respecto a la materia ambiental, concientizando al mundo sobre los problemas que han ido afectando nuestro planeta, por eso, nuestro país ha suscrito quince instrumentos internacionales de carácter vinculante sobre temas ambientales, además de múltiples declaraciones y planes de acción como la Agenda XXI o el Plan de implementación de Johannesburgo, acuerdos bilaterales y de cooperación regional, como el Acuerdo de Cooperación Ambiental de América del Norte, paralelo al Tratado de Libre Comercio. Los Estados Unidos Mexicanos forman parte de más de catorce organismos internacionales de cooperación para el desarrollo y la protección del medio ambiente, como el Programa de las Naciones Unidas para el Medio Ambiente o la Comisión de las Naciones Unidas sobre el Desarrollo Sostenible (Centro de 
Estudios Sociales y de Opinión Pública, 2006).

México ha firmado más de 77 acuerdos internacionales en materia de medio ambiente, de los cuales destacan: 1) Convenio de Estocolmo sobre contaminantes orgánicos persistentes; este convenio regula a los contaminantes orgánicos persistentes (COP) con propiedades tóxicas y son transportados por el aire, el agua y las especies migratorias. 2) Protocolo de Kioto; programa para desintoxicar el planeta, reducir la contaminación por gases tóxicos que causan el calentamiento global como son el gas metano, dióxido de carbono, y gases hexafluoruros. 3) Convenio de Viena; convenio con las naciones a modificar los efectos nocivos que genera la capa de ozono. 4) Convención de las naciones unidas sobre el cambio climático; enfocada a reducir las emisiones de gases contaminantes que elevan el efecto invernadero. 5) Carta mundial de la naturaleza; comprometía a los países firmantes a mantener la preservación del medio ambiente, el respeto por los distintos ecosistemas, disminuir la contaminación proveniente de las grandes industrias, conservar los recursos naturales y a idear estrategias auto sostenibles que ayuden a impulsar el desarrollo económico es los estados/provincias de cada país.

Ahora, es de reconocer que se requiere de la participación y coordinación de ambos órdenes de gobierno --federal y estatal-- para la óptima ejecución de acciones. Tanto la legislación estatal como la federal prevén la coordinación. La fracción tercera del artículo quinto de la Ley General de Equilibrio Ecológico y la Protección al Ambiente (2015, p. 6) señala que es de competencia federal "La atención de los asuntos que afecten el equilibrio ecológico en el territorio nacional o en las zonas sujetas a la soberanía y jurisdicción de la nación, originados en el territorio o zonas sujetas a la soberanía o jurisdicción de otros Estados, o en zonas que estén más allá de la jurisdicción de cualquier Estado;" así como el "El fomento de la aplicación de tecnologías, equipos y procesos que reduzcan las emisiones y descargas contaminantes provenientes de cualquier tipo de fuente, en coordinación con las autoridades de los Estados, el Distrito Federal y los Municipios; así como el establecimiento de las disposiciones que deberán observarse para el aprovechamiento sustentable de los energéticos;"

Por otro lado, la Ley Ambiental del Estado de Nuevo León $(2005$, p. 11) prevé que " $E l$ Estado y los Municipios ejercerán sus atribuciones en materia de preservación, restauración y conservación del equilibrio ecológico y la protección al ambiente, de conformidad con la distribución de competencias previstas en la Ley General, en ésta Ley y en otros ordenamientos aplicables. Los Municipios, en el ámbito de su competencia, podrán aplicar de manera supletoria lo dispuesto en esta Ley, a falta de reglamentación municipal específica en la materia".

Dicho lo anterior, la participación de la Federación resulta estratégica, principalmente en los siguientes asuntos:

\section{Contaminación Ambiental}

Al adentrarnos a la situación del estado, encontramos que se emitieron 8 millones 910 mil 110 toneladas de sustancias contaminantes durante 2017, posicionándose como la quinta entidad con mayor emisión total de contaminantes, después de Veracruz (19.95 mdt), Tamaulipas (17.37 mdt), San Luis Potosí (10.72 mdt) y Sonora $(9.28 \mathrm{mdt})$, de acuerdo con datos del Registro de Emisiones y Transferencia de Contaminantes (RETC) de la Secretaría de Medio Ambiente y Recursos Naturales (SEMARNAT) (Secretaría de Medio Ambiente y Recursos Naturales, 2017).

Las emisiones de bióxido de carbono representan el $99.68 \%$ de las emisiones de contaminantes de la entidad, seguido por otras sustancias como el metano $(0.20 \%)$ y el bióxido de nitrógeno $(0.12 \%)$. Hay que decir que las empresas de Generación de Energía Eléctrica emitieron más de la mitad de los contaminantes del estado (53.32\%), seguidas por las empresas Metalúrgicas (17.55\%), las Químicas (8.80\%), las de Cemento y cal (6.00\%) y las Automotrices (5.29\%). El $9.04 \%$ restante se emitió en sectores como petróleo y petroquímica, celulosa y papel, vidrio, entre otros.

Revista de Direito Brasileira | Florianópolis, SC | v. 26 | n. 10 | p. 230-242 | Mai./Ago. 2020 
La empresa Techgen S. A. de C. V., Central Eléctrica Pesquería, puesta en marcha en 2016, generó 2 millones 523 mil 512.72 toneladas de bióxido de carbono, equivalentes al $28.41 \%$ del CO2 emitido en Nuevo León durante 2017, de acuerdo con el reporte del RETC.

La Central Ciclo Combinado Huinalá, de la Comisión Federal de Electricidad (CFE) es la segunda más contaminante de la entidad, al producir $22.12 \%$ del bióxido de carbono del estado, seguida de Ternium México S. A. de C. V. (4.66\%) y Zinc Nacional S. A. (4.44\%).

\section{Pedreras}

Por otro lado, las pedreras que se encuentran en Nuevo León representan la industria más peligrosa y la que provoca más daños a la salud, son altamente contaminantes, pues desgajan los cerros esparciendo polvo en el ambiente de la ciudad ocasionando enfermedades respiratorias y cancerígenas. Además de esto, también se genera un ecocidio en las zonas donde operan por el uso de dinamita para extraer los recursos y deforestación.

Se ha detectado que la industria extractora de piedra caliza contribuye hasta en el 33 por ciento de aportación de partículas PM10 en el área metropolitana (Hernández, 2018b). De acuerdo al colectivo Únete Pueblo, hay alrededor de 30 pedreras piratas en Nuevo León y no existe regulación alguna (Hernández, 2018a).

La Procuraduría Federal de Protección al Ambiente (PROFEPA) señala que es facultad de competencia estatal la regulación, supervisión, inspección y vigilancia de las actividades que realizan las pedreras que operan en el estado de Nuevo León, no obstante, es una problemática en la cual el Gobierno Federal debe corresponsabilizarse.

Es importante mencionar que las pedreras no se encuentran incluidas en el artículo 111 BIS de la Ley General del Equilibrio Ecológico y la Protección al Ambiente (LGEEPA), la cual señala cuales son las fuentes fijas de jurisdicción federal; además que el funcionamiento de las pedreras en materia de impacto ambiental no está previsto dentro de lo señalado en el artículo 28 de la misma ley (Procuraduría Federal de Protección al Ambiente, 2018).

\section{Transporte urbano}

El transporte urbano en el estado de Nuevo León enfrenta una gran problemática respecto a servicio y precio. El Sistema Integral de Transporte Metropolitano (SITME) sufre de un deterioro día tras día, el parque vehicular utilizado como transporte urbano es altamente contaminante. El transporte público en muchos casos se encuentra en mal estado y sin mantenimiento lo que produce aún más contaminación.

La implementación del biodiésel en la red de transporte público permitiría que se mejore el aprovechamiento. Además de que tiene beneficios a nivel ambiental por la disminución de gases de efecto invernadero y a nivel mecánico porque brinda una mayor eficiencia en los autobuses. Por otra parte, al recolectar el aceite se evita que vaya al drenaje y genere problemas de contaminación del agua.

Las mejoras tecnológicas pueden contribuir a un transporte urbano más sostenible. Los combustibles más limpios, esquemas de inspección y mantenimiento, o formas de propulsión tales como la movilidad eléctrica, permitirían que el sistema de trasporte público de Nuevo León se volviera más eficiente, a la vez que se contamina menos.

\section{Parque vehicular}

Nuevo León es el $5^{\circ}$ estado del país con más parque vehicular con 2 millones 129 mil (INEGI, 2017), De los cuales 1 millón 593 mil son automóviles, 98\% de particulares; 8,700 
camiones para pasajeros, con porcentaje de $85 \%$ públicos y $15 \%$ particulares; 458,053 camiones y camionetas de carga, con alrededor del $95 \%$ de particulares, $4 \%$ públicos y 1\% oficiales y; 69,640 motocicletas de las que $99 \%$ son particulares. Es alarmante que un estado que concentra casi el 5\% del parque vehicular del país no tenga mecanismos que permitan hacerle frente a la contaminación provocada por los vehículos que usan combustibles fósiles, sobre todo si el $45.4 \%$ de las emisiones contaminantes en Nuevo León provienen del parque vehicular (Gobierno de Nuevo León, s.f.).

Al ser uno de los estados con más parque vehicular, el Estado Mexicano tiene que tener en cuenta que la contaminación del aire es, sin ninguna duda, un asunto de salud pública. Según estudios del ITESM (Grupo Legislativo PAN, 2018), la contaminación atmosférica del área metropolitana de Monterrey cada año les cuesta, al gobierno y a particulares, 800 millones de dólares (Gobierno de Nuevo León, s.f.). Entre atender problemas de salud provocados por la contaminación del aire y los costos de productividad por faltas de empleados enfermos.

\section{Verificación vehicular}

Los carros en Nuevo León contaminan $40 \%$ más en monóxido de carbono y 5 veces más en hidrocarburos que los de la zona metropolitana del Valle de México donde la verificación tiene más de 20 años (Gobierno de Nuevo León, s.f.).

La verificación no es una medida reciente. En más de una decena de ocasiones el gobierno de Nuevo León ha insistido en impulsar la Verificación vehicular en el estado, desde el año 2015, proyecto que implicaría un costo para los dueños de autos y que ha sido rechazado hasta por ecologistas (Hernández, 2017).

En 2018, Diputados del Congreso local aprobaron por mayoría la Ley de Hacienda del Estado para el 2019, que establece un aumento de $40 \%$ en el pago de refrendo de los autos, dinero que será destinado para la creación de la infraestructura para el programa de verificación vehicular (Padilla, 2018). Los legisladores informaron que se pretende recaudar 400 millones de pesos de este incremento, de los cuales 200 millones se destinarán para la generación de centros de verificación.

Los niveles de contaminación elevados provocan deficiencias en la calidad de vida, en términos de salud, igualdad social y reducción de impactos ambientales. Es el gobierno federal quien está obligado a prevenir y controlar la contaminación del aire, agua y suelo de acuerdo con el artículo $1^{a}$ de la LGEEPA. En consecuencia, es necesaria una regulación a nivel federal para la reducción de contaminación en la Zona Metropolitana de Monterrey.

El artículo $4^{\circ}$ de la Constitución Política de los Estados Unidos Mexicanos prevé la competencia de los tres niveles de gobierno conforme a lo siguiente, "la Federación, los Estados, el Distrito Federal y los Municipios ejercerán sus atribuciones en materia de preservación y restauración del equilibrio ecológico y la protección al ambiente, de conformidad con la distribución de competencias prevista en esta Ley y en otros ordenamientos legales."

Existen facultades relativas a materias o aspectos ambientales específicos, cuya atención le corresponde exclusivamente a la Federación, sin que los gobiernos de las entidades federativas y de los municipios puedan participar.

ARTÍCULO 5o.- Son facultades de la Federación: ...

...

XII.- La regulación de la contaminación de la atmósfera, proveniente de todo tipo de fuentes emisoras, así como la prevención y el control en zonas o en caso de fuentes fijas y móviles de jurisdicción federal..."

El Pleno de la Suprema Corte de Justicia de la Nación resolvió mediante la tesis jurisprudencial "PLANEACIÓN DEMOCRÁTICA DEL DESARROLLO NACIONAL Y SISTEMA NACIONAL DE DESARROLLO SOCIAL", que la planeación nacional de

Revista de Direito Brasileira | Florianópolis, SC | v. 26 | n. 10 | p. 230-242 | Mai./Ago. 2020 
desarrollo es la ordenación racional y sistemática de acciones que, con base en el ejercicio de las atribuciones del Ejecutivo Federal en materia de regulación y promoción de la actividad económica, social, política, cultural, de protección al ambiente y aprovechamiento racional de los recursos naturales, tiene como propósito la transformación de la realidad del país, de conformidad con las normas, principios y objetivos que la propia Constitución y las diversas leyes que las desarrollan establecen (Suprema Corte de Justicia de la Nación, 2009).

El Estado tiene interés en la defensa y mejoramiento de la seguridad pública, la imagen urbana, la salud pública, el medio ambiente y el buen manejo de las finanzas públicas, lo que implica una reconciliación entre el bienestar económico, los recursos naturales y la sociedad (Suprema Corte de Justicia de la Nación, 2018).

Por tanto, haciendo un ejercicio de hermenéutica convencional en estricta aplicación a los modelos de justicia constitucional en materia de Derechos Políticos, Económicos, Sociales y Ambientales (DESCA), concluimos y suscribimos lo planteado por los individuos de diversas organizaciones en el mundo, los cuales han propuesto diversas estrategias para la implementación del Desarrollo Sostenible, una de ellas es la Educación para el Desarrollo Sostenible (EDS) con el fin de que cada ser humano sea capaz de forjar un desarrollo sostenible y que a su vez el actor se encuentre preparado para prevenir y afrontar el cambio climático, la reducción del riesgo de desastres, pobreza y que tenga un consumo sostenible. Una de las intenciones de las EDS es que el ser individual sea capaz de adoptar decisiones colectivas que lo orienten a contribuir en pro del desarrollo comunitario sostenible. Por otro lado las EDS proponen el trabajo multidisciplinario para lograr su objetivo con la finalidad de que sean cubiertos todos los ámbitos posibles que se involucran en este tipo de desarrollo.

En la actualidad, Naciones como España, Reunión Unido o Alemania ya utilizan el enfoque de la EDS, para alcanzar la sostenibilidad, sin embargo aún ha faltado mucho por lograr ya que la educación ambiental aún no está en su mejor momento.

Por otra parte los países con un nivel industrial más alto requieren de un consumo más demandante, por lo que están generando un mayor daño al ecosistema mundial, ya que necesitan de un volumen de recursos naturales más abarcador para satisfacer sus necesidades económicas, sociales y energéticas

Las condiciones de los asentamientos humanos en muchas partes del mundo, en particular en los países en desarrollo, están deteriorándose principalmente como resultado de los bajos niveles de inversión en ese sector, atribuibles a las limitaciones globales en materia de recursos de esos países. Lo anterior requiere de una inversión constante en las comunidades rurales que dependen de las grandes ciudades, pero lamentablemente "En los países de bajos ingresos para los que se dispone de datos recientes, solamente un 5,6\%, en promedio, de los gastos del gobierno central se destinaban a vivienda, esparcimiento, seguridad social y bienestar”. (ONU, s.f., párr. 1)

Por tanto, el desarrollo comunitario sostenible en America Latina, por citar un caso; en Chile en donde se ha trabajado el desarrollo comunitario sostenible desde las políticas públicas y el análisis e implementación de las mismas; uno de los conceptos que se manejan dentro de dicho análisis es el de "enfoque de interfaz", este permite:

"observar las relaciones, la racionalidad y el poder que se juegan en la negociación de una política pública entre los gestores encargados de su implementación, las comunidades destinatarias en contextos dados y los contextos institucionales en los cuales estas experiencias se desenvuelven. Permite así analizar el engranaje, los lazos y redes que se desarrollan entre las partes, las expectativas y el rumbo que moldean la interacción entre estos actores." (Fernández y Robles, 2004)

En este mismo ámbito se encontró a Naturaleza y Cultura Internacional (NCI), esta es una 
organización que trabaja en pro del ambiente y la cultura como bien lo dice su nombre, desde 1997 ha buscado conservar las tradiciones ancestrales de los pueblos de la Amazonía, además de colaborar con comunidades pobres para establecer actividades sostenibles y generadoras de ingresos y al igual que la UNESCO se ha interesado por incentivar la educación ambiental.

"NCI desarrolla procesos de conservación vinculados siempre al desarrollo social, planificando sus actividades a largo plazo, más allá de la duración de los proyectos que se implementan en las áreas de trabajo. Estos procesos son planteados y ejecutados por profesionales locales, en base a las prioridades determinadas por las comunidades y gobiernos, creando una estrecha y fructífera relación de cooperación." (NCI, 2008).

Dicho argumentario enfocado a la obligatoriedad del Estado, el cual debe implementar políticas públicas de mejoramiento y conserva del medio ambiente, así como la pertinencia para que las personas puedan hacer efectiva y justiciable la tutela de sus derechos elementales, como lo es la salud, la propiedad y el libre desarrollo de la personalidad bajo los pilares de la economía y el desarrollo sustentable, lo que conlleva a una sinergia integral armonizada con los principios rectores del medio ambiente construidos desde el marco normativo mexicano y aplicándolo los tribunales constitucionales.

Dicho criterio lo comparte nuestro máximo tribunal, en el criterio jurisprudencial siguiente:

DERECHOS HUMANOS A LA SALUD Y A UN MEDIO AMBIENTE SANO. LA EFICACIA EN EL GOCE DE SU NIVEL MÁS ALTO, IMPLICA OBLIGACIONES PARA EL ESTADO Y DEBERES PARA TODOS LOS MIEMBROS DE LA COMUNIDAD.

La eficacia en el goce del nivel más alto de los mencionados derechos, conlleva obligaciones para el Estado, hasta el máximo de los recursos de que disponga; sin embargo, esa finalidad no sólo impone deberes a los poderes públicos, sino también a los particulares, pues la actuación unilateral del Estado resulta insuficiente cuando no se acompaña de conductas sociales dirigidas a la consecución de los valores que subyacen tras esos derechos, lo que implica que su protección sea una responsabilidad compartida entre autoridades y gobernados. Así, el medio ambiente sano, como elemento indispensable para la conservación de la especie humana y para el disfrute de otros derechos fundamentales, tiene carácter colectivo, porque constituye un bien público cuyo disfrute o daños no sólo afectan a una persona, sino a la población en general; por esa razón, el Estado debe implementar políticas públicas que permitan prevenir y mitigar la degradación ambiental, las cuales deben cumplir con estándares constitucionales y convencionales, además de contar con la participación solidaria de la comunidad, pues la salud se refiere a un estado completo de bienestar físico, mental y social, y no únicamente a la ausencia de enfermedad o incapacidad de las personas.

\section{SÉPTIMO TRIBUNAL COLEGIADO EN MATERIA ADMINISTRATIVA DEL PRIMER CIRCUITO.}

Rubro: "DERECHO FUNDAMENTAL A LA SALUD. IMPONE DEBERES TANTO A LOS PODERES PÚBLICOS COMO A LOS PARTICULARES QUE SE DEDICAN AL ÁMBITO DE LA SALUD.", publicada en el Semanario Judicial de la Federación y su Gaceta, Décima Época, Libro XVI, Tomo 1, enero de 2013, página 626.

Consideramos que de la revisión jurídica, es vital que esta Honorable Cámara de Diputados, exhorte al Poder Ejecutivo Federal a implementar acciones de regulación al parque 
vehicular, transporte público y entes contaminantes que coadyuven a implementar programas con el fin de reducción de contaminación en el Estado de Nuevo León. Así como en pleno respeto de la división de poderes, exhortar al Gobierno Federal y al Gobierno del Estado de Nuevo León para fortalecer los mecanismos de inspección en pleno respeto de sus facultades para reducir los índices de contaminación ambiental en la Zona Metropolitana de Monterrey.

Por lo anteriormente expuesto, resulta de imperiosa necesidad implementar acciones que, en su conjunto, contribuyan a mitigar los efectos negativos de la contaminación y deterioro del medio ambiente, principalmente en materia de movilidad y desarrollo económico.

\section{CONCLUSIONES}

Derivado de la reforma energética publicada el 20 de diciembre del 2013 en el Diario Oficial de Federación, se modifican los artículos 25, 27 y 28 de la Constitución Política de los Estados Unidos Mexicanos, y se crearon diversas dependencias con facultades para emitir, interpretar, aplicar y sancionar conforme a la regulación vigente del Sector Hidrocarburos.

Es bien sabido que, en la realización de las Actividades y Operaciones del Sector a lo largo de toda la cadena de valores, se pueden llegar a producir afectaciones al ambiente, a los ecosistemas, a las personas, a la población, a la economía nacional, etc.

Para velar por la conservación de una regulación funcional, es necesario realizar algunas adecuaciones a la legislación, toda vez que se ha detectado que existe una invasión de competencias a razón Estado y Federación, así como entre dependencias, detectando una excesiva sobre regulación dentro del Sector.

Sin embargo, el problema de la sobre regulación, se debe a que gran parte de la misma fue emitida con anterioridad a la reforma energética, y la regulación ya existente no fue modificada para ser acorde a las nuevas políticas regulatorias.

Para tratar de solucionar esto, se propone el proyecto "Reestructuración del Sector Energético", el cual busca, emitir un instrumento jurídico que sea capaz de delimitar las competencias y los alcances jurídicos de cada organismo o secretaria que interviene en las actividades del sector, ello a través del análisis detallado de los siguientes ordenamientos jurídicos.

Las actividades del Sector Hidrocarburos, cuentan con un ciclo de vida que comprende las etapas desde el Diseño, Construcción, Pre-arranque, Inicio de Operaciones, Mantenimiento, Cierre, Desmantelamiento y Abandono del sitio, estas actividades también deberán ser incluidas en el análisis de la regulación. (Podría existir una última etapa del ciclo de vida de un proyecto, la remediación, sin embargo, a través de la vigilancia en los procesos de las etapas anteriores, se busca evitar llegar la misma, sin embargo, también deberá ser materia del análisis).

Para lograr el objetivo del proyecto, se busca identificar en cada una de las Leyes y/o Reglamentos la problemática antes definida, con la intención de abarcar la omisión o aclarar la intención del legislador y así brindar a los regulados, permisionados o autorizados, una certeza jurídica y procedimental que sea viable, evitando la invasión de competencias.

\section{REFERENCIAS}

Centro de Estudios Sociales y de Opinión Pública (2006), "Contexto internacional", en Medio ambiente, disponible en: www.diputados.gob.mx/cesop/

Espinoza Canturin, E. Y. (2017). El proceso de establecimiento del área de conservacion regional "Bosque Montano de Carpish" y el rol de Naturaleza Y Cultura Huánuco.

INEGI (2017), “Parque Vehicular”, Disponible en: https://www.inegi.org.mx/temas/vehiculos/ 
Ley Ambiental del Estado de Nuevo León (2005), H. Congreso del Estado de Nuevo León, México.

Ley Federal de Metrología y Normalización (2009), Cámara de Diputados del H.Congreso de la Unión, México.

Ley General del Equilibrio Ecológico y la Protección al Ambiente (2015). Cámara de Diputados del H. Congreso de la Unión, México.

Ley de Hidrocarburos (2016), Cámara de Diputados del H. Congreso de la Unión.

Ley de Órganos Regulados Coordinados en Materia Energética (LORCME) (2014), Cámara de Diputados del H. Congreso de la Unión, México.

Fernández, Margarita y robles, Claudia (2004), La dinámica de Intefaz de Procesos de Reforma del Estado, Descentralización y Democratización en el Espacio Local: Dos Estudios de Casos. Informe de Investigación para el Proyecto de Desarrollo Comunitario Sustentable en Perspectiva Comparada. Center for Latin American Socia Policy. Claspo. Universidad de Texas. Chile.

Gobierno de Nuevo León (s.f.), "Mitos y realidades de la verificación vehicular”, Consultado el 23 de febrero, 2019 en: http://www.nl.gob.mx/mitos-y-realidades-de-la-verificacion-vehicular

Grupo Legislativo PAN (2018), "Verificación vehicular gratuita", (Consultado, 24 de febrero de 2019 en: http://www.hcnl.gob.mx/glpan/2018/01/verificacion-vehicular-gratuita.php) Hernández, Iram (2018b) “El colmo... hay pedreras pirata en Nuevo León”, El Horizonte. Consultado el 23 de febrero de 2019: http://www.elhorizonte.mx/local/el-colmo-hay-pedreraspirata-en-nuevo-leon/2192483

Hernández, Iram (2018a), “Gobierno de NL insiste con imponer verificación vehicular”, El Horizonte, consultado el 23 de febrero, 2019: http://www.elhorizonte.mx/local/gobierno-de-nlinsiste-con-imponer-verificacion-vehicular/2111004

Hernández, Iram (2017), "Pedreras contaminan 10 veces más de lo que informa el estado". Noticias Info 7. Consultado el 23 de febrero de 2019: http://www.info7.mx/locales/pedrerascontaminan-10-veces-mas-de-lo-que-informa-estado/1992013).

ONU (s.f.), 7. Fomento del desarrollo sostenible de los recursos humanos. Párr. 1. Disponible en: https://www.un.org/spanish/esa/sustdev/agenda21/agenda21spchapter7.htm

Padilla, Jesús (2018), "REGIOS PAGARÁN VERIFICACIÓN VEHÍCULAR CON AUMENTO EN EL REFRENDO”, Reporte Indigo, consultado el 23 de febrero de 2019:

https://www.reporteindigo.com/reporte/regios-pagaran-verificacion-vehicular-con-aumento-enel-refrendo/

Procuraduría Federal de Protección al Ambiente (2018), "La regulación y vigilancia de las actividades de las pedreras en Nuevo León no es competencia de la PROFEPA, sino del gobierno del estado", Informe. Gobierno de México. Disponible en: https://www.gob.mx/profepa/prensa/la-regulacion-y-vigilancia-de-las-actividades-de-laspedreras-en-nuevo-leon-no-es-competencia-de-la-profepa-sino-del-gobierno-del-estado 
Suprema Corte de Justicia de la Nación (2009), Novena Época, Gaceta del Semanario Judicial de la Federación Tomo XXX, página 1543, Pleno, tesis P./J. 76/2009; PLANEACIÓN DEMOCRÁTICA DEL DESARROLLO NACIONAL Y SISTEMA NACIONAL DE DESARROLLO SOCIAL.

Suprema Corte de Justicia de la Nación (2018), Décima Época, Gaceta del Semanario Judicial de la Federación Tomo II, libro 56, Plenos de circuito, Tesis: PC.III.A. J/49 A (10a.); LICITACIÓN PARA LA PRESTACIÓN DEL SERVICIO DE MANTENIMIENTO Y MEJORA DE ALUMBRADO PÚBLICO MUNICIPAL. NO PROCEDE OTORGAR LA SUSPENSIÓN PROVISIONAL AL PARTICIPANTE NO FAVORECIDO EN EL PROCEDIMIENTO RELATIVO CUANDO SE SOLICITA PARA EL EFECTO DE QUE NO SE FORMALICE Y EJECUTE EL CONTRATO DE CONCESIÓN.

Secretaría de Medio Ambiente y Recursos Naturales (2017), "Informe del Registro de Emisiones y Transferencia de Contaminantes (RETC)". Consultado el 24 de febrero de 2019 en: http://sinat.semarnat.gob.mx/retc/retc/index.php 